\title{
DIE ROLLE FAMILIENGESCHICHTLICHER HINWEISE IN DER SUPERVISION
}

(Erhalten: 2. September 2007; angenommen: 24. Oktober 2007)

In der Supervision wird mit und an Erfahrungen aus dem Berufskontext gearbeitet. Sie ermöglicht dem Klienten eine jeweils besondere, individuelle Art des reflektierenden Erfahrungslernens. Sie zielt auf die Stärkung der Berufspersönlichkeit, auf Veränderung in der Berufspraxis durch Lernen ab. Die Supervision als gemeinsamer Reflexionsprozess beruht auf dem Lernengagement des Supervisanden und geht davon aus, dass das Entscheidungsrecht über die einzelnen Schritte auch beim Supervisanden liegt. Der Supervisor hat dabei im Prinzip nicht das Recht, über die beruflichen Situationen, die von der jeweiligen Person in die Supervision eingebracht werden, hinauszugehen. Manche beruflichen Probleme, Schwierigkeiten oder Konflikte (Kompetenzunsicherheit, Hemmungen und Blockaden in bestimmten Arbeitssituationen, Konflikte mit Kollegen/Kolleginnen und Vorgesetzten usw.) können jedoch einen unbearbeiteten persönlichen Hintergrund haben und auf frühere Sozialisationserfahrungen oder sogar auf Ereignisse oder Momente in der Familiengeschichte zurückgeführt werden. Diese emotional mitschwingenden Erinnerungen aus der persönlichen Lebensgeschichte oder aus der über mehrere Generationen hinweg wirkenden Familiengeschichte (z. B. Stigmatisierungserfahrungen, Familienmythen, Ängste, Verbote, Befehle, „Botschaften“) beeinflussen oft unbewusst die Wahrnehmungs- und Reaktionsmuster des Supervisanden, führen zu „blinden Flecken“ und damit zur undifferenzierten und unkontrollierten Vermischung der beruflichen und persönlichen Sphäre bei ihm. Besonders dann, wenn sie implizit in Form von Metaphern, Symbolen, Geschichten usw. zum Ausdruck kommen oder angesprochen werden, dürfen sie nicht ausgeblendet, sondern sie können und sollen zum Gegenstand der Reflexion gemacht werden. Dabei ist aber die Gefahr unbedingt zu vermeiden, dass der Prozess über die Grenzen der Supervision hinausgeht und sich zur Therapie entwickelt. An einem Einzel- sowie Teamsupervisionsprozess soll aufgezeigt werden, wie durch die Erschließung, Bewusstmachung und Integration

* Eszter Sz. Kováts, IBS International Business School, Tárogató út 2-4., H-1021 Budapest, Ungarn; eszkov@ibs-b.hu 
dieser auto- oder familienbiographischen Bezüge die „berufliche Selbsterfahrung“ gesteigert und damit die Berufsidentität gestärkt werden kann.

Schlüsselbegriffe: Supervision, Einzelsupervision, Gruppensupervision, Teamsupervision, Lebensgeschichte, Familiengeschichte, Erfahrungslernen, Berufspersönlichkeit, „berufliche Selbsterfahrung"

The Role of Family History References in Supervision: Supervision aims at grouping and processing experiences obtained in a professional context. It provides the client with a unique, personal method to reflexive experience based learning. Its goals are to strengthen the professional personality, and to change the specific professionalism through learning. Supervision, as a common reflection process, is based on the commitment of the supervised person to learning, and the assumption that the supervised person is able to make specific decisions regarding individual steps. In theory, the supervisor has no right to exceed the professional situations brought under supervision by the given person. However, behind some professional problems, difficulties or conflicts (uncertainties of competence, inhibitions or blocks appearing in certain work situations, conflicts with peers or superiors, etc.) there may lie unprocessed threads, which may be tracked to earlier socialisation experiences or even events or incidents in family history. Originating from personal history or the multi-generation history of a family (e.g. family beliefs, fears, stigmatisation experiences, prohibitions, commands, identity experiences, 'messages') these memories often even unconsciously influence the perception and reaction patterns of the supervised, and may lead to the establishment of 'blind spots', and thereby the undifferentiated and uncontrolled coalescence of the personal and professional spheres. We may and should especially react to these if they become implicitly expressed during supervision in the shape of metaphors, symbols, stories, etc. However, while doing so one should definitely avoid the danger of exceeding the limits of supervision, and thereby transforming the process into therapy. An individual and a group supervision process serve to illustrate how it is possible to contribute to the increasing of 'professional self-awareness' and thereby the strengthening of professional identity by uncovering, increasing awareness of and integrating these personal or family history threads.

Keywords: supervision, individual supervision, group supervision, personal history, family history, learning through experience, professional personality, 'professional self-awareness'

\section{Die persönlichen Ebenen in der Supervision}

Supervision baut auf beruflichen Erfahrungen auf und macht sie zugleich zum Gegenstand der Reflexion. Supervision besteht in der Wahrnehmung der im Spiegel der Reflexion aufleuchtenden Erkenntnisse: im Lernen. Das längerfristige Ziel besteht in der Aufdeckung, Bewusstmachung und Integration der jeweils besonderen, individuellen Art des Erfahrungslernens: Indem wir Erfahrungen sammeln, stützen wir uns auf diese, wenden sie auf einer neuen Ebene an und machen bei ihrer praktischen Anwendung erneut Erfahrungen - der niederländische Supervisor Louis van Kessel bezeichnet dies als „Lernen des Lernens“. Über all dies hinaus zielt sie ab auf die Stärkung der mit ihrem wahren Wesen identischen, reifen Persönlichkeit im beruflichen Feld, auf die Veränderung durch Lernen. 
In der Supervision wird mit Erfahrungen aus dem Berufskontext gearbeitet. Der Supervisor hat nicht das Recht, über die beruflichen Situationen, die von der jeweiligen Person in die Supervision eingebracht werden, hinauszugehen. Lernen und damit Veränderung wird immer individuell aufgebaut und gestaltet. Die Veränderungsenergien sind tief im Ich-Kern der jeweiligen Person zu finden und zu mobilisieren. Die Gefühle und Lebenserfahrungen können uns zu ihnen führen. Durch Supervision wird der Erkenntnis und dem Lernen Raum eröffnet und freigehalten. Die Entscheidung über die Art und Weise und das Ausmaß des Lernens liegt ausschließlich beim Supervisanden. Das Mitgehen des Supervisors mit der Entwicklung des Lernengagements des Supervisanden beruht auf der Anerkennung des Entscheidungsrechts des Supervisanden über die notwendigen Schritte und wird durch den mehrfach auf den Prozess zugeschnittenen Vertrag ermöglicht (ZIMMERMAN et al. 1986).

Ich hatte mich einmal mit einem Kollegen - einer vielseitigen Persönlichkeit unterhalten. Er erzählte mit großer Leidenschaft von seinen Erfahrungen aus seiner langjährigen, beeindruckenden beruflichen Vergangenheit. Die Synthese seiner Erfahrungen war - wie ein fein geschliffener Kristall - funkelnd klar. Er hatte vor, seine Gedanken in einem Aufsatz schriftlich zusammenzufassen. Die persönlichen Erfahrungen selbst, die als Grundlage für diesen Aufsatz dienten, wollte er dagegen als etwas in eine wissenschaftliche Studie nicht Passendes weglassen. Ich dachte darüber nach und kam zu dem Schluss, dass der Aufsatz mit seinem strengen und engen Rahmen als eine hermetisch geschlossene Vitrine zwar die wahren Schätze der Wissenschaft bewahren wird. Wird aber der Betrachter der Kristalle auch die Möglichkeit haben, über die faszinierende Ausstrahlung der glänzend klaren Kanten und Lichter der Kristalle hinaus auch den Zauber der verborgenen Farben - der lebendigen Abdrücke der Wirklichkeit - erleben zu können? Ist es immer ein Gewinn für uns, wenn ausschließlich reine Theorie - als Essenz der Erfahrung - vermittelt wird? Geht mit den als ,nicht dazugehörig“ angesehenen persönlichen Erfahrungen häufig nicht auch ein großes Maß an „Lebendigkeit“ verloren?

In Supervisionssitzungen ist es oft ein schwieriger Weg, von der Ebene der Gedanken (Erklärungen, Analysen, Deutungen) zu den wahren inneren „Abdrücken“ der Erfahrung zu gelangen und aus diesen wie aus Bausteinen eine - immer tiefer greifende und gleichzeitig immer weiter ansteigende - „Spirale“ zu bauen, die die sich ständig erweiternde, flexible, jedoch stabile Bahn des selbstreflexiven Lernens der Alltage bestimmen kann. Für mich bedeutet Supervision diesen Weg - ein gemeinsames Bauen, ein gemeinsames Schaffen, in dem der Supervisand sowohl über den Bauprozess und die Bauphasen als auch über das Bauwerk selbst disponiert. Der Supervisor hat - meines Erachtens - in erster Linie die Aufgabe und die Verantwortung, dem Klienten gegenüber eine achtsame, annehmende und unterstützende Haltung einzunehmen - und zwar ohne jegliche Nebentöne. Ebenso dazu gehört die mitgehende, über das Sichtbare hinausgehende und hinausweisende Aufmerksamkeit und das auf die beiden Richtungen der Spiralbewegung fokussierende, perspektivische Sehen und „Sehenlassen" sowie die Schaffung eines psychologischen Lernraums, in dem der Supervisand befähigt wird, sein berufliches und damit verbundenes persönliches Erlebnismaterial gleichzeitig mit einer Freiheit zu betrachten, die das Verstehen und die Aufnahme 
von neuen Impulsen zulässt und sich in einem über das Verstehen hinausgehenden und zu neuem Leben erweckenden Erahnen zu verweilen.

Im vorliegenden Aufsatz werde ich die Bedeutung bestimmter, aus der primären Sozialisation stammender Erlebnisinhalte für die Supervision aufzuzeigen versuchen. Dabei geht es um Kindheitserlebnisse, die zugleich als Ereignisse aus der (sogar über mehrere Generationen hinweg wirkenden) Familiengeschichte oft nicht nur die Menschen-, Welt- und Situationssicht bestimmen, sondern - als mehrfache „Abdrücke“ in der Erinnerung - auch den Selbstwahrnehmungs- und Lernhorizont des Supervisanden beeinflussen, und daher auch auf das persönliche und berufliche Leben des Supervisanden in großem Maße einwirken. Diese tief eingebetteten „Abdrücke“ neigen stark dazu, in den Fokus zu geraten, und halten den Supervisanden - wie in einer „Doppelbindung“ - in einer stark frustrierenden Spannung gefangen. Als würden sie stark pulsierendes Licht ausstrahlen, tauchen sie immer wieder für einen Augenblick auf; bevor sie jedoch dem Blick, dem Verständnis oder gar der Intuition zugänglich wären, verschwinden oder verändern sie sich dermaßen, dass sie für den Beobachter unkenntlich werden. Und auf diese Weise tauchen sie immer wieder auf und verschwinden dann wiederholt. Dieses Pulsieren kann im Supervisanden Unruhe, fallweise aber auch eine für das supervisorische Lernen förderliche Ambivalenz auslösen. Angesichts dieser Ambivalenz bin ich der Ansicht, dass der Supervisor in diesen Fällen eine vermehrte Verantwortung trägt - sowohl für den Lernprozess des Supervisanden als auch für die eingesetzten Interventionen. In diesen Augenblicken braucht der Supervisor vor allem seine Sensibilität: sein Gefühl für Grenzen und seine zur Selbstreflexion fähige Empathie, die ihm die differenzierte Wahrnehmung und Aufnahme von vielschichtigen Botschaften ermöglicht, ferner seine Kreativität, seine Ressourcen zur Auseinandersetzung und vor allem seine Zurückhaltung ermöglichende Ruhe und Fähigkeit zur Verfolgung von verschiedenen parallel ablaufenden Prozessen.

In Zusammenhang mit der Supervision taucht immer wieder die Frage auf, wo die Grenzen zwischen Therapie und Supervision liegen. Die therapeutischen Effekte der Supervision sind zwar allgemein bekannt und anerkannt, die Frage der Grenzen gibt jedoch in vielen Fällen in Expertenkreisen Anlass zu heftigen Debatten. Die historische Beziehung zwischen Psychotherapie und Supervision gilt zwar als Fakt, reicht aber als ausschließliche und vorrangige Erklärung für die Gemeinsamkeiten zwischen der auf Erfahrungslernen basierenden Supervision und der Therapie nicht aus. Die Persönlichkeit kann theoretisch nach Belieben in verschiedene (z.B. berufliche und persönliche) Teile differenziert werden. Die Struktur der Persönlichkeit besteht in der Tat aus verschiedenen Schichten: Diese reichen von den tiefen Schichten der archaischen, der prä-, peri- und postnatalen Wirkungen und der ersten Objektbeziehungserfahrungen über die Leistungen der Primär-, Sekundär- und Tertiärsozialisation bis hin zu den an verschiedene Erwachsenenrollen gebundenen. Es steht auch nicht zur Frage, dass die durch die Rollenausübung erwünschten Verhaltensweisen eine mehrdimensionale (emotionale) Einbezogenheit der Persönlichkeit fordern. 
Die Chronologie, Intensität und die Wirkungen der Erinnerungen aus unserer persönlichen Lebensgeschichte bilden die Grundlage für unsere subjektive Weltanalyse... „Unsere“ Wahrheit oder „unsere“ Realität entsteht dadurch, dass wir unsere persönliche Geschichte kontinuierlich auf unsere unmittelbaren Erfahrungen daraufsetzen oder übertragen.

(LAVEMAN 1995)

Es ist zu beachten, dass das „,berufliche Ich“ kein neues Gebilde des Erwachsenenalters ist, sondern eine Form des ,persönlichen Ich“ im beruflichen Kontext darstellt (REAMS 1995). Dementsprechend kann wirkliche Veränderung (Entwicklung) nur mithilfe eines komplexen Ansatzes herbeigeführt werden.

Wir vermögen Personen, Situationen, kulturelle Systeme und sogar uns selbst nur durch die verfestigten Verzerrungen unseres Wahrnehmungs- und Beziehungssystems wahrzunehmen. „Unsere Berufspersönlichkeit“, die sich bei der Ausübung unseres Berufs präsentiert, gestaltet sowohl ihre interpersonalen als auch interkulturellen Interaktionen und Beziehungen in diesem begrenzten Referenzrahmen. Wir alle bewegen uns im Rahmen eines persönlichen Referenzsystems, der in der Folge der Umwelterfahrungen auf unserem Lebensweg entsteht und unser Verhalten, Motivationen und unsere Urteilsbildung ebenso beeinflusst. Dieses Referenzsystem wird auf den verschiedenen Ebenen der Sozialisation bestätigt, wobei der Schule durch die reflexive Darstellung der historischen Entwicklung unserer Zivilisation eine besondere Bedeutung zukommt. All dies kann verdeutlichen, dass auch Supervision, die auf die Förderung der Berufspersönlichkeit abzielt, nicht effektiv sein kann, wenn dieser Bezugsrahmen nicht erweitert und dessen geheime Winkel - „blinde Flecken“ - mithilfe von Bewusstmachung und Einsicht nicht beleuchtet werden. Die Bearbeitung der persönlichen Bezüge stellt aber weder eine Aufgabe noch eine Chance für die Supervision dar. Dies gilt umso mehr, weil der Supervisor von seiner Ausbildung her mit anderen Schwerpunkten arbeitet. Die Supervision als Arbeitsform weist ein Zielsystem und Rahmenbedingungen auf, die grundverschieden sind von denen der therapeutischen Arbeit zugrunde liegenden Zielsetzungen und Rahmenbedingungen.

\section{Lebensgeschichtliche Bezüge in der Supervision}

Die entscheidende Rolle der familiären Sozialisation bei der Berufswahl und beruflichen Sozialisation sowie ihr Einfluss auf die Ausübung der Berufsrolle sind in der Berufspsychologie wohlbekannt. Dementsprechend finden sie auch in der Supervision besondere Beachtung.

M. van der LINDEN (1987) definiert den dreidimensionalen Gedanke-Gefühl-Verhalten-Ansatz als grundlegende Regel in der supervisorischen Bearbeitung. Die dabei auftauchenden Sozialisationserfahrungen werden von ihr als ein wichtiger Bezugspunkt erachtet, in dessen Licht die besondere, individuelle Verknüpfung des persönlichen und beruflichen Ich und deren jeweils individuelle Gesetzmäßigkeiten und Muster wahrnehmbar sowie deren Wirkungen interpretierbar werden. Als wichtiges Kriterium für die erfolgreiche Supervision bezeichnet R. WELTER-ENDERLIN (1991), die in ihrem Werk das Erfahrungsmaterial der persönlichen Lebensgeschichte als gespeicherten 
Vorrat an Mustern und Mitteln betrachtet, die Förderung der Sensibilität. Aus ihrer Sicht hat die Supervision nicht nur die Freiheit, sondern auch die Aufgabe, dieses Erfahrungsmaterial (das durch Resonanzen bzw. in Form von Metaphern und Geschichten zum Ausdruck kommt) aufzudecken, der kreativen Anwendung zugänglich zu machen, zu mobilisieren und in das berufliche Repertoire zu integrieren. Sie gibt als eine der Zielsetzungen von Supervision an, dass durch sie die berufliche Tätigkeit intensiver, lebendiger, sozusagen spielerischer wird und damit neue Sichtweisen und neue Formen des Umgangs mit Situationen beim Supervisanden zustande kommen.

Ich würde gern diese Wirkung (nämlich wie die Erkenntnis von autobiographischen Bezügen zur Veränderung des Umgangs mit Berufssituationen und dadurch der inneren Atmosphäre einer Einrichtung führt) an einem Teamsupervisionsprozess veranschaulichen. Es wurde von einer kurz vor der Supervision umstrukturierten Einrichtung mit geringem Personalstand Supervision für das gesamte Mitarbeiterteam beantragt. Die Supervision sollte dabei dazu dienen, den Hintergrund der für die berufliche Tätigkeit hinderlichen Mitarbeiterkonflikte zu klären. Auf die Frage hin, welches aus ihrer Sicht das wichtigste Merkmal des Einrichtungsprofils wäre, gibt die Leiterin der Einrichtung - nennen wir sie einmal Ria - in der dritten Supervisionssitzung folgende Antwort: „das Helfen“. Sie bekommt aus der Gruppe die Frage: „Was bedeutet für dich Helfen?" „Geben und bekommen“, antwortet sie darauf, „Sicherheit, Schutz und Menschlichkeit“. Sie macht eine Zeichnung als Symbol für dieses Bild: Eine alte, gebückte Oma am Gehstock und ein kleines Mädel spazieren Hand in Hand. Sie zeigt der Gruppe die Zeichnung und erinnert sich an ihre Kindheit zurück, als sie eine Zeitlang gerade in der Umgebung gewohnt hat, wo jetzt die Einrichtung steht. Als kleines Mädchen hatte sie regelmäßig zur Aufgabe, eine alte Frau aus der Nachbarschaft, die auch mit dem Gehstock nur schwer gehen konnte, täglich bei ihrem Spaziergang zu begleiten. „Ich habe oft das Gefühl, meine eigenen Wurzeln unter den Füssen zu haben", sagt Ria, an deren Leiterhaltung von den Kollegen früher gerade kritisiert wurde, dass , sie die Einrichtung fast wie ihr eigens behandelt“. Durch die neuartige Perspektive des Gefühls ,auf den eigenen Wurzeln zu gehen“ entdeckt Ria in ihrer Arbeit als Leiterin ein der Eifersucht ähnliches Gefühl und eine Art ständiges Zweifeln daran, ob ihre Kollegen auch dann gute Arbeit leisten, wenn sie abwesend ist. Zu ihrer Pflegearbeit sagt sie Folgendes: „Als wäre das ganze Neubaugebiet, das hier 'gewachsen' ist, meiner Obhut anvertraut." Während der Erzählung benennt sie mehrere Probleme, die aus dieser neuen Perspektive von ihrer Rollenvermischung und zu starken emotionalen Verstrickung herzurühren scheinen. Dabei entdeckt sie, dass sie ihre pflegerische Identität derzeitig noch stärker wahrnimmt als ihre Leiteridentität, und sie sich mit der Leiterrolle bzw. mit den neuen Aufgaben in Zusammenhang mit der fachlichen Leitung des vor kurzem erweiterten Teams nicht ausreichend identifizieren konnte. Sie empfindet eine starke Neigung dazu, am liebsten alles selbst zu machen. Erst in der Supervisionssitzung wird ihr bewusst, dass sie noch kein wirkliches Vertrauen zu ihren Kollegen entwickelt und bisher auch nicht viel dafür getan hat. Sie hat ihre Kollegen zwar akzeptiert, aber sie war nicht darum bemüht, sie besser kennen zu lernen. In der Supervisionssitzung wird ihr starkes Interesse an den Wurzeln anderer und ihre starke Orientierung an diesen Informationen in der Herstellung der persönlichen 
Beziehung zu den Klienten bewusst: Sie fragt nach diesen Informationen, sie merkt sie sich und weist auch in den Gesprächen immer wieder auf sie hin. Parallel dazu fällt ihr wiederum auf, dass sie als Leiterin nie an der Familie und den Wurzeln ihrer Mitarbeiter interessiert war. Ein so geartetes Interesse ihren Kollegen gegenüber würde - ihrer eigenen Formulierung zufolge - tatsächlich ihre Anteilnahme an den Kollegen beweisen. Ihre Erkenntnis in der Sitzung stimmte mit der Distanzerfahrung ihrer Kollegen und dem Anspruch des Teams überein, die Mitarbeiterbeziehungen persönlicher zu gestalten. Die Fokussierung auf diesen gemeinsamen Punkt führte zu gegenseitigem Verständnis und zu einer konstruktiven Wende im Team: Es wurden Pläne zur Veränderung der erkannten Situation entwickelt, in denen auch dem Gespräch über die Herkunft und Familientraditionen Raum gegeben wurde. Es wurden auch einige gemeinsame Programme außerhalb der regulären Arbeitszeit vereinbart und organisiert. Gleichzeitig wurden in den Teamsitzungen auch Fragen bezüglich der Einrichtung und Arbeitsorganisation thematisiert und besprochen. Dank dieser Entwicklungen kam es zu einer besseren Anwendung der inneren Ressourcen sowie zu einer geklärten Aufgabenteilung.

\section{Bedeutung familienbiographischer Bezüge für die Supervision}

Wie auch die oben dargelegte Teamsupervision illustriert, knüpfen sich die in der Supervision auftauchenden persönlichen Erlebnisinhalte meistens an die durch die beruflichen Situationen unbemerkt berührten Kindheitserinnerungen und an die von ihnen herrührenden und bis zur Gegenwart wirkenden Einstellungen und Wahrnehmungs- und/oder Reaktionsmuster.

In der Supervision wollen nicht selten über mehrere Generationen hinweg wirkende Momente aus der Familiengeschichte (z. B. Familienmythen, Ängste, Stigmatisierungserfahrungen, Verbote, Befehle, Identitätserfahrungen, „Botschaften“) zutage treten, die nicht unmittelbar zum persönlichen Lebensweg gehören. Sie können jedoch das Berufserleben, Berufsbewusstsein sowie berufliches Rollenverhältnis und Rollenverhalten des Supervisanden grundsätzlich bestimmen. Über ihre Existenz, ihren Einfluss oder Macht scheint der Supervisand meistens nichts zu wissen. Wenn er doch etwas über sie weiß, kommen sie ihm selbstverständlich oder unveränderbar vor. Wenn familiengeschichtliche Momente als implizite Inhalte im Supervisionsprozess auftauchen, zeigt dies den Anspruch auf die Herstellung oder Stärkung der Beziehung zu den eigenen Wurzeln. Dies gibt Auskunft darüber, dass die innere Entwicklung in Richtung der persönlichen Integrität nach einem Ausgleich oder Abbau der übertriebenen Identifikation mit der Berufsrolle verlangt. Nach PÜHL (1994) ist die Rollenidentifikation „ein Versuch des Ich, mit den gesellschaftlichen Widersprüchen umzugehen". Er bezieht sich dabei auf eine These von Parin, der die Identifikation als einen „Fremdkörper“ im Ich betrachtet. Parin bezeichnet dies als ein „Symptom“, dessen „Gewinn dem sekundären Krankheitsgewinn ähnlich ist“, und kommt zu dem Schluss: Um seine eigenen ursprünglichen Bedürfnisse erkennen und Schritte in Richtung von deren Befriedigung machen zu können, muss das Ich durch die Lösung von Rollen- 
idealen und Rollenanforderungen von der Ich-Struktur seine Autonomie zurückgewinnen, auf die „Befriedigungsformen durch die Rolle“ verzichten und stattdessen die für die Autonomie förderlichen inneren Kräfte einsetzen.

Die Erinnerungen aus der Familiengeschichte, die Familienmythen und Familiennormen (ebenso wie die Sozialisationserfahrungen oder die - auf einer anderen Ebene des Selbstausdrucks liegenden - psychosomatischen Symptome), die überwiegend in Form von leichten Andeutungen oder beiläufigen Bemerkungen erscheinen, besitzen also einen besonderen Signalcharakter für die Supervision. Sie signalisieren Parallelen zwischen den (inneren) beruflichen und persönlichen Konflikten und verdeutlichen gleichzeitig auch die notwendige Richtung des nächsten Schrittes in der inneren Entwicklung.

Der nächste Ausschnitt aus einem Supervisionsprozess kann besonders anschaulich machen, wie die familiengeschichtlichen Fäden wie „ein Kompass“ die Entwicklungsrichtung zeigen können, sowie als Beispiel für eine achtsame Schrittfolge dienen, in deren Rahmen diese Fäden durch die Bearbeitung der beruflichen Erfahrungsinhalte und emotional mitschwingenden Erinnerungen aus der persönlichen Lebensgeschichte ins Bewusstsein gehoben werden.

Mária hat ihren Supervisionsprozess im Anschluss an eine Gruppensupervision auf Vorschlag ihrer Leiterin hin am Arbeitsplatz selbst initiiert. Zur Zeit der Supervision war sie als Wirtschaftsberaterin tätig. Ihre umfangreiche Klientel reichte von Direktoren von Großunternehmen über die Leiter von kirchlichen und staatlichen Organisationen bis zu Einzelunternehmern. In ihrer Arbeit erzielte sie hervorragende Erfolge, sie war selbstständig und einfallsreich. Sie war an ihrem Arbeitsplatz anerkannt, sie hielt sich jedoch für erfolglos. Sie sprach oft von ihrem Wunsch, („einmal“, wenn sie es sich wird leisten können) mit den rationalen und geschäftsmäßigen Tätigkeiten aufzuhören und sich ganz der karitativen Arbeit zu widmen. In die Supervisionssitzungen hat sie Situationen in Zusammenhang mit folgenden grundlegenden Themengruppen gebracht:

- Kompetenzunsicherheit

- Hemmungen und Blockaden in Verhandlungssituationen (vor allem „einem bestimmten Typ“ von männlichen Leitern gegenüber)

- Konflikte mit (überwiegend männlichen) Kollegen und ihrer Vorgesetzten

- Ambivalente Gefühle bezüglich ihrer eigenen beruflichen Erfolge.

Sie zeigte in den Supervisionssituationen große Betroffenheit. Sie hat an den hier angesprochenen Fragen auch in der Zwischenzeit, also zwischen den einzelnen Sitzungen weiter gearbeitet. Sie kam einmal sehr aufgeregt zur Supervisionssitzung hereingestürzt. Eine Verhandlungssituation von vor einigen Tagen beschäftigte sie sehr intensiv. Sie geißelte sich wütend wegen ihres „ungeschickten“ und „lächerlichen“ Verhaltens. Sie hatte eine Besprechung mit einem Kunden, einem Direktor, der ihr undurchschaubar und unnahbar vorkam. Sie sah sich ziemlich ausgeliefert und wehrlos; der Direktor schien sie nicht ernst zu nehmen und seine Position zu missbrauchen. „Ich war verlegen und begann herumzualbern“ - sagte sie. Und dann fuhr sie in der Erzählung fort: „Ich wurde immer nervöser und geriet immer mehr in Verlegenheit. Ich spürte, dass ich sogar mich selbst auch nicht ernst nehmen könnte." Dann fragte 
sie mich nach kurzem Schweigen: „Wie soll ich mich dem Menschentyp annähern, mit dem ich keinen gemeinsamen 'Faden' finden kann?" Ich greife gerne die durch Betonung, Lautstärke, andere metakommunikative Begleiterscheinungen oder Wiederholung verstärkten Wörter oder Sätze auf und hebe sie durch „Verstärkung“ hervor. Auch diesmal habe ich das getan. Nun boten sich mehrere Möglichkeiten an. Ich entschied mich für den mehrmals und in mehreren Formen benutzten Ausdruck „verlegen“: „Du sagtest, du wärest verlegen gewesen, in Verlegenheit geraten...“ „Ja, stimmt", antwortete sie, ,diese ganze 'Verlegenheit': als FRAU in der Welt zu sein.“ Sie dachte für eine Weile nach, dann fügte sie noch Folgendes hinzu: „Das ist so kompliziert. Ich meine, man kann eigentlich nie genau wissen, was richtig wäre.“ Wie sie davon sprach, zeichneten sich ihre Ängste vor den Männern ab, die, wie sie sagte, „der Frau eine Falle stellen“, „die Frau in eine Falle locken“. Sie erzählte all dies mit einer ungewöhnlichen Fülle von emotional beladenen, fast archaisch anmutenden Bildern, während ich bisher gerade ihre übertriebene Rationalität als einen bremsenden Faktor empfunden hatte. Dieser plötzliche Wechsel gab dem Affekt, mit dem sie über all dies sprach, einen besonderen Akzent. Der Affekt selbst kam mir bereits bekannt vor, da er schon mehrmals bei der Erwähnung von konfliktbeladenen Personen (Leiterin, bestimmte Verhandlungspartner, Kollegen) aufgetaucht war. Dieser plötzliche Fokuswechsel und die reibungslose „Passung“ des neuen Bildes („Falle") zu den auch die Arbeitssituationen begleitenden Affekten machte mich darauf aufmerksam, dass wir hier für einen Augenblick stehen bleiben sollten. Ich dachte, dass wir unbedingt dafür sorgen müssen, diese beiden (oder eventuell sogar mehrere) Schiffe, die auf dem gleichen Ozean schwankend dümpeln, voneinander zu trennen. Ich war mir darüber im Klaren, dass die Gefahr wirklich besteht, dass der Prozess über die Grenzen der Supervision hinausgeht und sich zur Therapie entwickelt. Ich wollte das unbedingt vermeiden. Und nicht nur aus dem Grunde, weil dies ,ein unsachgemäßer Schritt" oder nicht durch unseren Kontrakt geregelt wäre. (Das wäre sonst natürlich auch ein ausreichender Grund gewesen.) In diesem Fall lag aber der entscheidende Grund dafür darin, dass dies dieselbe undifferenzierte und unkontrollierte Vermischung der beruflichen und persönlichen Sphäre bedeutet hätte wie dies bei Mária - meiner Einschätzung nach - den Ausgangspunkt für ihre beruflichen Schwierigkeiten gebildet hatte. Während mir diese Gedanken durch den Kopf gingen, schaltete Mária inzwischen schon um: Die Affekte verbanden sich mit einem relativ neuen, in die Biographie eingebetteten Auslöser - mit den Erinnerungen an die dramatische und stürmische Ehe und Scheidung von Mária. Ich sah ihr an, wie sie vom Strom der aus der Tiefe emportauchenden Schmerzen mitgerissen und über das flammende Feuermeer begrabener Erinnerungen getrieben wird und wie sie Zeit, Raum und konkrete Situation hinter sich lässt. Dort und dann hatte ich keine Möglichkeit, einen neuen Kontrakt zu schließen, den früheren weiter zu präzisieren und genauer zu umreißen. Die „Verschiebung“ - oder mit dem Begriff von Laveman die „,isomorphische Abbildung“ - war schon eingetreten. Als Mária von dieser anstrengenden inneren Reise zurückgekehrt war, fragte ich sie, ob sie eine Ähnlichkeit zwischen den wachgerufenen Erinnerungen und den sie begleitenden Emotionen und der geschilderten Verhandlungssituation sehen würde. Verblüfft entdeckte sie an sich selbst - mit 
ihren Worten gesagt - die gleichen Spuren der „Brandwunden“, welche auch das als peinlich empfundene Treffen mit dem Direktor, mit dem sie keinen ,gemeinsamen Faden“ fand, hinter sich gelassen hatte. „Die früheren Wunden sind tiefer eingebrannt und haben ewige Spuren in mir hinterlassen“, sagte sie anschließend.

Zum Schluss der Sitzung haben wir alles, was wir in diesen 90 Minuten erlebt haben, gemeinsam durchdacht, und einen neuen, weiteren Rahmen für die folgenden Supervisionssitzungen vereinbart. An diesem Tag verabschiedete sich Mária von mir mit den folgenden Worten: „Die heutige Sitzung hat mich sehr tief berührt. Ich hätte nie gedacht, dass die Ereignisse meines Lebens auch in meiner Arbeit eine so wichtige Rollen spielen (könnten)!“

In der nächsten Sitzung berichtete sie von einem körperlichen Symptom: Sie hatte sowohl am Abend nach der voriges Mal als Fall bearbeiteten Verhandlungssituation wie nach der Sitzung selbst starkes Nasenbluten. Und zwar ein so starkes wie in den Monaten nach der Hochzeit und dann später während der Scheidung. Sie verknüpfte das Symptom - aufgrund der in der Supervisionssitzung erkannten und bewusst gewordenen Zusammenhänge - selbst mit ihrer damaligen und ihrer jetzigen Situation.

In der bisher geschilderten (aus dem mittleren Drittel des Gesamtprozesses herausgegriffenen) Phase der Supervision haben die in die Supervision gebrachten Situationen aus dem Berufsalltag unbearbeitete, traumatische Ereignisse aus dem frühen Erwachsenenalter des persönlichen Lebenswegs und die mit ihnen verbundenen Gefühle in die Gegenwart geholt: Jene Gefühle (und die dann während der Bearbeitung hervortretenden Erinnerungen, die diese Gefühle verursacht haben), die die berufliche Situation „nicht in Ruhe gelassen“, sondern immer wieder provoziert und aufgewühlt haben und dadurch zu Blockierungen und „Kurzschlüssen“ in der Fähigkeit zum Umgang mit beruflichen Situationen geführt haben. Die in die Familiengeschichte zurückreichenden Fäden, die Márias Umgang mit beruflichen Situationen auf eine subtile Weise beeinflussten, konnten erst später, nach einem Überblick und der Bewusstmachung der betroffenen Erfahrungen auf dem persönlichen Lebensweg bzw. nach der Auflösung der daraus resultierenden fachlich-persönlichen Verstrickungen zutage treten.

Nach einigen Sitzungen tauchte das Thema FRAU-Sein wieder auf. Ausgangspunkt war das Gefühl der Erfolglosigkeit von Mária, das sie immer nach der monatlichen Bewertung der Arbeitsleistung ergriffen hatte. Márias Leistung fand zwar wieder Anerkennung, sie selbst aber schämte sich dafür und begann ihre Fehler aufzuzählen. Sie war sehr unruhig und angespannt. Sie geißelte sich mit maßloser Autoaggression. All ihre heftigen inneren Affekte strömten aus und erfüllten das Zimmer. Es war fast spürbar, wie die Strömung dieser Affekte den Blick zunehmend trüb machte. Nicht nur den Blick von Mária, sondern parallel dazu auch meinen Blick. Die Züge, Gesten, die ganze Körperhaltung und Atmung zeigten die gleiche besessenheitsartige typische Ruhelosigkeit wie beim Wendepunkt in der vorher geschilderten Supervisionssitzung. Ich wollte diese dynamische Kraft nicht bremsen. Ganz im Gegenteil: Ich spürte gerade, dass hier das Auftauchen eines entscheidenden Faktors bevorsteht, und dass es für den Schritt nach vorn (zur tieferen Einsicht) wichtig ist, jetzt diesen Sturm zu 
durchleben. Aber ich bestand gleichzeitig unbedingt darauf, dass ihr (uns) dabei ein Orientierungspunkt zur Verfügung steht. Ich fragte sie, ob sie die Gefühle, die durch sie schwirrten, benennen könnte, und bat sie anschließend darum, das für sie bestimmende Gefühl auszuwählen. Sie hob das Gefühl der „Demütigung“ hervor. Ich versuchte ihrer Antwort nachzuspüren und ihr auf dem Weg der inneren Fokussierung zu folgen, und auf meine Intuition hörend dem Wort laut nachzuspüren: „Demütigung . . . Du empfindest Demütigung . . . Etwas ist in dir gedemütigt worden ... 'DIE FRAU'“, erwiderte sie. Sie schluchzte mehrmals in mehreren Wellen tief auf.

Die beinahe traditionelle Erwartungsstruktur ihrer Familie tat sich in der Sitzung auf, derzufolge ,eine ganz normale, anständige Frau einen Ehemann finden kann, der sie zu ernähren vermag“. Eine Frau, die etwas wert ist, „bleibt mit der Familie zu Hause und wird von ihrem Mann unterhalten“. Die Aufgabe der Frau besteht darin, „ihren Mann zu höheren Leistungen anzuspornen“. „Denn eine Frau darf auf keinen Fall wehrlos sein!", wiederholte sie die Worte ihrer Großmutter, die damals ihren Mann und Familie mit strenger Hand gesteuert hatte. Márias Mutter hatte sich noch an diese Familiennorm gehalten und zwar als „Unterhaltene“, aber unter schweren finanziellen Verhältnissen gelebt. Mária hörte sie noch oft darüber klagen, wie „wehrlos“ sie sich in dieser Situation fühlte. Ihre Mutter spricht jedoch bis heute wie von einer misslungenen Situation davon, dass ihre - zur Zeit der Supervision bereits fast 50 Jahre alte - Tochter berufstätig ist und gut verdient. „Sie haben nie an mich geglaubt“, beschwerte sich Mária. „Mária kann das nicht schaffen“wurde schon in ihrer Jugend, aber besonders seit ihrer Scheidung (als sie wegen der Erkrankung ihres Mannes und dann infolge der Scheidung gezwungen war, eine Arbeit anzunehmen) und wird auch bis heute oft fast wie ein geflügeltes Wort in der Familie wiederholt. In den Supervisionssitzungen sagte sie wie zu sich selbst und zu ihrer Großmutter folgende Worte: „Ich habe versucht, meinen Mann anzutreiben, aber vergebens!“ „Das bedeutet es, gedemütigt zu sein“, sagte sie nach kurzem Schweigen, „eine berufstätige Frau, die niemand unterhalten will, ist keine richtige Frau. Sie ist erniedrigt." Dann setzte sie fort: „Es entstand Widerstand und Abwehr... Und sie spielen auch heute in jeder Situation stark mit." Sie erinnerte sich an die von Generation zu Generation weitergegebenen ,guten Ratschläge“ und Mythen ihrer Familie, die von der „vernünftigen Herstellung der Sicherheit“ und „Bekämpfung des Egoismus der Männer“ handelten.

Als weiteres Moment aus der Familiengeschichte tauchte noch in dieser Sitzung die Geschichte der Tante der Großmutter mütterlicherseits auf, die nach der Geburt ihres außerehelichen Kindes gezwungen war, als Dienstmädchen zu arbeiten, um sich selbst und ihr Kind ernähren zu können. Das wurde von der Familie nicht nur verurteilt, sondern als ein ihr aufgebürdetes Schandmal erlebt. Letzlich führte Mária das familiäre Schreckensbild von Demütigung und Wehrlosigkeit auf dieses Ereignis zurück: „Sie galt als Schandfleck der Familie, sie war die 'Missgeburt'." Sie entdeckte eine Parallelität zwischen sich selbst und dieser Verwandten aus früheren Zeiten: „Ich befinde mich in der gleichen Situation. Ich bin auch eine alleinerziehende und berufstätige Frau.“ Nach kurzer Pause fügte sie noch hinzu: „Das ist eine Falle! Ist demzufolge eine Frau in jedem Fall ausgeliefert und gedemütigt? Wird ihr immer das gleiche Geschick zuteil - egal, ob sie arbeitet oder unterhalten wird?" Und dann später sagte 
sie noch: „Heute habe ich auf jeden Fall auch die Tränen meiner Mutter, Großmutter und vielleicht sogar meiner Urgroßmutter vergossen."

Nachdem wir unseren Blick auf die Arbeitssituationen zurückgelenkt hatten, fand Mária mehrere Zusammenhänge zwischen den persönlichen und beruflichen Situationen. Über die in die jeweilige Supervisionssitzung eingebrachten Situationen hinaus verwies sie auch auf weitere Themen, die entweder in früheren Sitzungen angesprochen oder aber gar nicht thematisiert worden waren, zurück. Sie fand die neu entdeckten Zusammenhänge offensichtlich sehr gewichtig. In Zusammenhang mit dem konkreten Supervisionsthema hob sie Folgendes hervor: Erfolg und positive Feedbacks sind äußerst wichtig für sie, da sie für sie zugleich als eine Art „Bestätigung“ gelten. Gleichzeitig mit der Anerkennung und Betonung ihrer großen und guten Leistungen aber „beginnt“ ihr das Brandmal dieser Familienschande - das der „zur Arbeit genötigten, gedemütigten und wertlosen Frau“ - „zu brennen“ und sie empfindet Scham und Wut. An dieser Stelle sagte sie: „Das schrecklich Störende daran ist, dass die Gefühle zuweilen in mir herumwirbeln. Sie lassen mich nicht einmal Freude empfinden. Dann werde ich immer mehr sauer. Auch jetzt spüre ich diese Wut.“ „Du spürst wieder diese Wut", wiederholte ich ihre Worte. „Diese Wut ist wieder da.“ „Ja, sie ist da“, antwortete sie. „Sie ist wieder da, aber sie gehört nicht hierher . . Sie ist wie ein ungebetener Gast.“ Und dann nach kurzer Pause fuhr sie fort: „Hier weiß ich, dass sie nicht hierher gehört, dort aber kann ich sie nicht isolieren."

$\mathrm{Zu}$ meinen Lieblingswerkzeugen gehört ein mehrteiliges Achatstein-Set, ergänzt durch einige andere Halbedelsteine sowie Fluss- und Seesteine, -muscheln und -schnecken. Sie sind nach Farbe, Form, Größe und Muster äußerst unterschiedlich. Ich legte sie vor Mária hin. Ich fragte sie, ob sie Lust dazu hätte, mithilfe dieser Steine die Vielfalt der Gefühle, die sie bei der Anerkennung am Arbeitsplatz gleichzeitig empfindet, zu vergegenwärtigen. Ihre Augen strahlten. Sie fing gleich an die Steine zu sortieren. Ich legte ein kleines verchromtes Tablett mit den Steinen auf ein Tischlein. Sie arbeitete zuerst auf dem Tablett. Dann nahm sie die Steine vom Tablett und legte sie auf den Tisch. Mit einer plötzlichen Bewegung legte sie es auf den Boden und sagte als Erklärung etwa Folgendes: „Das Ganze ist ja nicht so glänzend.“ Als sie mit der Auswahl fertig war und die Steine als Gefühle identifizierte, bat ich sie, durch die Anordnung auch die Beziehungen unter den einzelnen Gefühlen darzustellen. In der Anordnung waren die freudvollen Gefühle über die Anerkennung ihrer hervorragenden Leistung - wie z.B. Freude, Stolz, Stressentspannung nach angestrengten Zeiten, Vorfreude auf neue Ambitionen - von den belastenden, destruktiven Emotionen, die aus der persönlichen Sphäre hierher übertragen werden, voneinander klar zu unterscheiden. Dann zeigte sie auf die Letzteren und sagte: „Das ist hier mein Reisebündel." Sie brachte damit zum Ausdruck, dass ihr Leben so viel leichter wäre, wenn sie all diese Gefühle in einem Bündel isoliert bewahren und selbst regulieren könnte, wann sie hervortreten sollen. Schließlich kam es zu einer symbolischen Umsetzung dieser Idee: Sie packte die Steine, die für negative Gefühle standen, in ein Taschentuch ein, und mit verlegenem Kichern suchte sie für das Päckchen eine dunkle Ecke auf dem Regal. Hier nutzte ich die Gelegenheit, die Therapie als eine eventuelle Option für sie anzusprechen und ihre diesbezüglichen Bedenken und Fragen mit ihr 
gemeinsam zu durchdenken. Das „Reisebündel“ blieb bis zum Ende des Supervisionsprozesses auf dem Regal liegen. Mária nahm es nur ein einziges Mal als Symbol hervor, aber wickelte es nicht aus. In der letzten Sitzung antwortete sie auf meine Frage, was nun wohl mit dem „Bündel“ geschehen sollte, dass wir es auswickeln können, weil die im Verlauf der Supervision entdeckten negativen Gefühle „bereits auch in ihr isoliert vorhanden sind“, auch wenn sie häufig erst nachher wahrnimmt, dass „das Päckchen schon aufgegangen ist"“.

In den folgenden Supervisionsssitzungen gerieten die Paralellitäten zwischen dem persönlichen und beruflichen Ich schon oft und blitzschnell in den Fokus. Zum Abschluss des Prozesses nannte Mária gerade ihre diesbezügliche neue Erkenntnis als die größte Lernerfahrung für sie.

Wie es den geschilderten Fallbeispielen aus der supervisorischen Praxis zu entnehmen ist, zeugt das Auftauchen von familiengeschichtlichen Bezügen in der Supervision, die sich aus irgendeinem Grund in einer gegebenen Berufssituation manifestieren, von dem umfassenden und zutiefst persönlichen Wunsch nach einer inneren Neuordnung. Ihr Ausgangspunkt ist oft eine persönliche Erfahrung in einer beruflichen Situation, die durch ein vertrautes Schema zutage tritt und die dann auf der Ebene der Selbsterfahrung (Reflexion) durch die Aufdeckung und Erfassung dieses konkreten Schemas bewusst gemacht werden soll. Falls dies aktuell und notwendig ist, „geschieht" es einfach: Das Signal kommt an und falls es freie Bahn (d.h. Aufmerksamkeit) bekommt, entfaltet es sich dank seiner auf die Veränderung gerichteten inneren Energien wie von selbst. Der Supervisor braucht keine Motivationstechniken oder besondere supervisorische Interventionen einzusetzen, es genügen die verständnisvolle, annehmende und aufnehmende Anwesenheit, die das Hören und das gemeinsame Fortschreiten ermöglicht, sowie die Sicherung der Möglichkeit für den Supervisanden, sich zu jeder Zeit - auf seine eigene Art und Weise und nach seinem Bedarf von dieser Anwesenheit überzeugen zu können. Als eminentes Qualitätsmerkmal dieses gemeinsamen Fortschreitens gilt der psychologische Raum, in dem sich Supervisor und Supervisand im Rahmen eines gegenseitigen Annäherungsprozesses aufeinander einstellen. Dadurch können sie dem Erfahrungskern, der auch die Energien zur inneren Veränderung als komplexes und grundlegendes Informationsbündel enthält, näher kommen. Eine unabdingbare Voraussetzung für diesen Prozess ist das gemeinsame Finden des Ausdrucksmittels und des Tempos, das der Sprache und dem Rhythmus der inneren Fragestellung und Erfahrungsbearbeitung am meisten entspricht. Oft ist es die Metasprache der Symbole und verdichteten Hinweise, die in diesem selbstreflexiven Dialog die zur Einleitung der Veränderung notwendige, neuartige Zugangsweise sichern kann; hier geht es um Symbole, die durch die Addition ihrer allgemeinen und persönlichen Bedeutungsinhalte die Veränderungsenergien zu mobilisieren vermögen.

Um dieses Sich-Aufeinander-Einstellen und die zurückhaltende, dem anderen freien Raum gebende Haltung auf das erforderliche Niveau einzustellen, braucht der Aufnehmende (Supervisor) nicht nur ein unendlich feines Proportionsgefühl, hohe Wachsamkeit und Konzentration, sondern gleichzeitig auch die Fähigkeit zur inneren Entspannung als Voraussetzung für die Aufnahmefähigkeit, die Offenheit für die Verschmelzung in einem „gemeinsamen Atmen“. Gerade deswegen schreibt PÜHL (1994), 
dass die für die „,berufliche Selbsterfahrung“ förderliche Entwicklung in der vertrauteren, personzentrierten Form der Einzelsupervision in tiefere Schichten einzudringen vermag als es bei Gruppensupervisionsformen der Fall ist. In der Einzelsupervision engt sich aber - wie das auch von Pühl festgestellt wird - der Interaktionsspielraum in bedeutendem Maße ein, wodurch nicht nur die Intensität der dynamischen Kräfte geringer, sondern auch die Grenze zwischen Therapie und Supervision undeutlicher wird. Daraus ergibt sich, dass das Maß und Verhältnis von Annäherung und Distanzierung in der Einzelsupervision eine entscheidende Rolle spielt und dieses Maß und Verhältnis nur durch eine fein vibrierende Abbildung der augenblicklichen Verhältnisse angemessen reguliert werden kann. Dazu soll der Supervisor eine spezifische Haltung - eine parallele Wahrnehmung mit einem Doppelfokus - einnehmen, die ihm ermöglicht, ein „Oszillogramm“ zu seiner Orientierung zu erstellen, das durch die Wahrnehmung der im Hier und Jetzt vergegenwärtigten zukünftigen Veränderung den inneren Prozess des Supervisanden und des Supervisors gleichzeitig anzeigt. Dies ist der von GENDLIN (1989), WiLTSCHKO (1996) und KöHNE \& WILTSCHKO (1986) beschriebenen unterstützenden, aber nicht-eingreifenden Haltung ähnlich, mit der sowohl die Fokussierung als auch die fokussierende Person begleitet wird.

Die Summierung der durch die - durch den Status innerhalb der Familie geprägte - jeweils spezifische Erlebniswahrnehmung aufgenommenen Impulse kann zu einer Energieanhäufung führen, die - wenn sie in der Supervision zutage treten kann und auch beachtet wird - dem Supervisanden helfen kann, über die „Schutzmauer“ der Berufsrolle hinauszublicken und ihre bisher eventuell als grausamer Abgrund wahrgenommene - und über die Person hinausweisende - wahre Identität zu entdecken.

Wenngleich die Einzelsupervision dem Individuum mehr Raum und Freiheit lässt und sie mehr dazu anregt, die persönlichen Fäden offener und tiefer in die Persönlichkeit zu integrieren, so kann dieser Anspruch auch in den verschiedenen Formen der Gruppensupervision nicht außer Acht gelassen werden. Die Gruppensettings (Gruppen- und Teamsupervision) bieten den Teilnehmern natürlich einen anderen Zugang und ein anderes Maß dazu an. Deren Realisierung verlangt vom Supervisor - über die Einstellung auf den Supervisanden hinaus - eine Wachsamkeit und ein Gefühl für Grenzen, die ihm auch die Wahrnehmung von Ansprechbarkeit, Belastbarkeit und Lernweg der Gruppenteilnehmer ermöglichen. Einen entscheidenden Faktor stellen dabei die gegenseitige Diskretion in der Gruppe, das Maß und die Stabilität der Gruppenintimität und der Grad des „Miteinanders“ der Gruppe dar. Die Gruppe selbst stellt eine autonome Entität mit besonderen Ansprüchen, Bedürfnissen, Sensibilität und Belastbarkeit dar, die der Supervisor als eine eigene Einheit und samt dem fokussierten Supervisionsprozess als ungebrochene Ganzheit wahrzunehmen und zu behandeln hat. Diese bestimmen vor allem die Art und Weise, Form und Tiefe, mit der die auftauchenden persönlichen Erlebnisinhalte angegangen werden dürfen. In den Gruppensupervisionsformen erweitert sich „der interaktive Spielraum“ (PÜHL 1994) in bedeutendem Maße, die Dynamik wird lebhafter und die Reflexionsbasis vergröBert sich.

Sowohl auf der Oberfläche als auch in der Tiefe verläuft eine Vielzahl von Selbsterfahrungs-, Reflexions- und Selbstreflexiosnprozessen parallel zueinander. Lernen 
wird multidimensional. In der Gruppe ist eine gleichzeitige und sich gegenseitig verstärkende Entwicklung in der am Fall arbeitenden Person, in den anderen Gruppenmitgliedern, in der Gruppe als Ganzem und im Supervisor zu beobachten. Das Verfolgen all dieser Faktoren erfordert seitens des Supervisors auch eine verstärkte selbstreflexive Aufmerksamkeit und eine mehrdimensionale und parallele Reflexion.

Diese „Dichte“ des psychischen Raums enthält ein energetisches Plus, das verdichtete Formen sowohl der Äußerung als auch der Aufnahme, Bearbeitung und des Verstehens hervorruft bzw. anbietet. Gerade deshalb kann die symbolische und interaktive Interpretation in der Gruppensupervision gegebenenfalls einen besonderen Akzent bekommen und sie vermag erschütternde Umwälzungen zu induzieren. Die funkensprühenden Ausbrüche von Entwicklungsenergien sowie die aktive Aufnahme, Begleitung und zugleich Reintegration der die Veränderung einleitenden und begleitenden Katharsis im Supervisionsprozess (beispielsweise über diesbezügliche Reflexionsfragen, die nicht den Supervisanden, sondern auch die Gesamtheit der Gruppe betreffen) erfordern vom Supervisor eine „verdichtete Präsenz“. In der Teamsupervision kommt dem Schutz der Grenzen des persönlichen und beruflichen Ich eine noch größere Bedeutung zu als bei anderen Formen der Supervision. Die persönlichen Erfahrungen, die trotzdem zum Vorschein kommen wollen, können nur einen sicheren und für die Entwicklung förderlichen Rahmen erhalten, wenn der Supervisor mit dem Supervisanden streng im beruflichen Kontext bleibt und bei der Bestimmung von Ausmaß des Austauschs der hier gewonnenen Reflexionen die Gruppenbalance und die Entscheidungsfreiheit der betroffenen Person in höchstem Grade beachtet.

Die Supervisionsverfahren, die Selbstreflexion in den Vordergrund stellen, können durch das Strömenlassen von inneren Erlebnissen und Bildern, mittels Übertragungen und „Entwürfe“ - in Anknüpfung an berufliche Rollenmomente - die persönlichen und somit auch die Sozialisations- und familiengeschichtlichen Erlebnisinhalte berühren und fallweise beleben. Diese blitzen in der Supervisionssituation oft nur als Signale auf, manchmal aber bedürfen sie einer konkreteren Erscheinung. Sie haben ihren Platz in der symbolischen Erschließung genauso wie in der verbalen Reflexion. Wichtig ist es beim Auftauchen von solchen Inhalten, diese zu integrieren, und in dem Fall, wenn der supervisorische Rahmen und Kontext nicht ausreichen, die Notwendigkeit der Fortsetzung der Bearbeitung in einem anderen Förderrahmen (z. B. Selbsterfahrung oder Therapie) offen anzusprechen und dem Betroffenen bewusst zu machen.

\section{Zusammenfassung}

Der vorliegende Aufsatz untersucht Platz und Stellenwert von Sozialisationserfahrungen und besonders von familiengeschichtlichen Erfahrungen im Supervisionsprozess. Dabei wird von der Annahme ausgegangen, dass das persönliche und familienbiographische Erlebnismaterial, das in der Supervision zum Ausdruck kommt, einen Signalwert besitzt. Es markiert einen Entwicklungspunkt, bei dem zur Beantwortung von inneren beruflichen Fragen und zur Weiterentwicklung der (beruflichen) Persönlich- 
keit die Erschließung, Bewusstmachung und Integration der mit ihnen verbundenen und eventuell bis in tiefere Schichten durchdringenden persönlichen Erfahrungen unverzichtbar ist. Der Aufsatz nimmt die Voraussetzungen, Möglichkeiten und methodischen Bezüge für die Supervision aus der Sicht der Supervision unter die Lupe.

\section{Referenzen}

GENDLIn, E.T. (1989) Életproblémák megoldása öneröből: „Fókuszolás”, Übers. K. VARGA (Budapest: Tankönyvkiadó).

KÖHne, F. \& J. WiltschKo (1986) Focusing: Des Körpers eigene Psychotherapie [Videoband und Heft] (Garmisch-Partenkirchen: Mediateam Psychologie).

LAVEMAN, L. (1995) 'The Multi-Level Supervision Model and the Interplay Between Clinical Supervision and Psychotherapy', The Clinical Supervisor 12:2 (Jan) 75-91.

Linden, M.v.d. (1987) 'Socialisatie in Supervisie' Supervisie in opleiding en beroep, 4:2, $12-20$.

PÜHL, H. (1994) 'Einzelsupervision' in H. PÜHL, Hrsg., Handbuch der Supervision (2. Aufl.; Berlin: Marhold) 85-95.

REAMS, R. (1995) 'Selfobject Transferences in Supervision', The Clinical Supervisor, 12:2 (Jan) 57-73.

Welter-Enderlin, R. (1991) 'Zur Bedeutung der Kontextsensibilität in der systemischen Supervision', in H. BRANDAU, Hrsg., Supervision aus systemischer Sicht (Salzburg: Otto Müller) 261-67.

WiltschKo, J. (1996) Von der Sprache zum Körper (Würtzburg: DAF).

Zimmerman, N., L.E. Collins \& J.M. BACH (1986) 'Ordinal Position, Cognitiv Style, and Competence: A Systemic Approach to Supervision', The Clinical Supervisor 4:3 (Aug) $7-24$. 